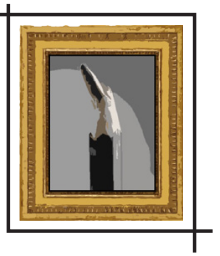

\title{
LA INTERVENCIÓN EDUCATIVA A TRAVÉS DE LA EDUCACIÓN A DISTANCIA EN CENTROS PENITENCIARIOS
}

\section{Yhomara Martí González*}

Resumen: En este artículo se presenta una explicación de la importancia que ofrecen los principios de la intervención y abordaje educativos para el modelo de educación superior a distancia en los centros penitenciarios, pues a ellos no les es ajena la formación universitaria valorándose que la presencia de esta población reclusa y madura en las universidades con toda su experiencia puede ser un valioso ingrediente enriquecedor de la vida universitaria que no debe desaprovecharse.

Palabras clave: Educación a distancia. Centros penitenciarios. Educación. Educación Superior. Intervención.

La sociedad actual se ha hecho más exigente en cuanto a las demandas de conocimientos y destrezas que exige a sus ciudadanos. Un mundo de cambios acelerados requiere de nuevos aprendizajes y la posibilidad de disponer de múltiples saberes alternativos. Dentro de esta sociedad que se desarrolla le corresponde un papel importante a la educación con todo lo que ella representa sobre todo en lo relacionado con la inclusión; al respecto Viegas Fernández (2002, p. 3) afirma:

La educación debe contribuir a disminuir progresivamente las desigualdades sociales, acabar con el hambre, erradicar el analfabetismo, reequilibrar ecológicamente el planeta, terminar con las guerras, poner punto final al racismo, a la xenofobia, al sexismo y todas las demás formas de discriminación, promover una cultura de amor, ayudar a lograr una existencia-vivencia humana con sabiduria.

La educación es un proceso dialéctico cuyo resultado será el formar al hombre para la vida, en toda su complejidad:

\footnotetext{
* Especialista en Docencia Universitaria. Profesora de la Universidad de Granma, Cuba. Investigadora en temas de Educación en Centros Penitenciarios. E-mail: kalil@usp.br
} 
Educar es depositar en cada hombre toda la obra humana que le ha antecedido; es hacer a cada hombre resumen del mundo viviente, hasta el día en que vive; es ponerlo a nivel de su tiempo, para que flote sobre él y no dejarlo debajo de su tiempo, con lo que no podrá salir a flote; es preparar al hombre para la vida (MARTí PÉREZ, 1961, p. 14).

Dado el carácter plural y multifacético de la educación es que se explica la diversidad de intervenciones educativas que se pueden realizar en diferentes escenarios de la sociedad. El concepto de intervención educativa no es nuevo pero sí en años recientes se ha hecho un amplio trabajo intelectual en torno a él. Este concepto estaría ligado a satisfacer necesidades, a la búsqueda de entendimientos, a satisfacer necesidades axiológicas, es decir valores que se estarían educando a lo largo del proceso de intervención; este parecer en particular implicaría varias acciones de carácter pedagógico, psicológico y sociológico.

El centro penitenciario no debe escapar de aplicar diferentes intervenciones educativas si tenemos en cuenta el tipo de población y educación a desarrollar en este escenario, separado de toda política, pues como establecimiento de reclusión y cumplimiento de las penas no puede escapar a esa concepción. Él debe conformar un hábitat en el que garantizándose las funciones de reclusión y custodia se creen espacios interiores en los que sea posible el cumplimiento de ese fin esencial de la institución con los resultados más beneficiosos posibles desde la privación de libertad para la vida en libertad.

A la luz de las anteriores distinciones, se puede volver la mirada hacia algunos principios que posee la intervención educativa dentro de los establecimientos penitenciarios, si tenemos en cuenta palabras como: diseñar, planificar, gestionar, desarrollar y evaluar, los principios sirven de base para establecer el tipo de intervención educativa a emprender. En nuestro trabajo creemos que estos principios deben estar dirigidos a:

- La intervención educativa debe ser inclusiva;

- Debe tener acciones para un aprendizaje participativo y desarrollador;

- Clima educativo adecuado teniendo en cuenta el escenario donde se desarrollen las acciones;

- Sistema de evaluación y autoevaluación diseñados correctamente.

La inclusión para la educación en los momentos actuales constituye un reto. Se trata de llegar a unos estudiantes en el que existen varias diferencias en cuanto a origen social, económico, cultural, sexo, religión, pensamiento, aptitudes físicas y psicológicas, capacidades, intereses, expectativas, ritmos de aprendizajes, valores a desarrollar y penas jurídicas a cumplir. La inclusión implica que el estudiante se adapte a lo que exige la educación; él debe desarrollar capacidades que le permitan formarse proponiéndose objetivos desde la propia situación en que se desarrollan. Será necesario, además, atender a la diversidad de estudiantes 
que estamos formando; por estas razones se ponen en práctica estrategias metodológicas que permiten que estos estudiantes aprendan de forma individual y grupal mediante actividades complejas utilizando diferentes recursos humanos y tecnológicos.

El segundo principio se basará en la necesidad de un cambio en la propia concepción del aprendizaje participativo y desarrollador con el fin de promover el desarrollo integral del estudiante, motor impulsor en aspectos como las capacidades, destrezas, habilidades, actitudes. Los estudiantes deben participar, en primer lugar, en la toma de decisión de incorporarse al programa o no, de manera que, de alguna forma, sea parte de una decisión personal, fruto de la reflexión, el diálogo, el consenso y el compromiso personal.

El estudiante que participará en programas educativos dentro del establecimiento penitenciario con determinadas características - de las cuales se hablará más adelante - deben saber que son los máximos responsables de su propio proceso educativo, y que sin su implicación no es posible avanzar hacia las metas propuestas, por ello el proceso de participación debe ir acompasado con el compromiso personal, una especie de acuerdo o contrato que especifique en qué términos se va a dar esta implicación, hasta dónde y en qué proceso. Deberá cumplir con las demandas del perfil del profesional y su desarrollo personal, participando de una manera activa en este proceso; ellos con su experiencia van descubriendo qué papel juegan dentro de este, teniendo en cuenta conocimientos previos de manera que puedan conectar lo nuevo con lo ya conocido, produciéndose una reestructuración de este conocimiento previo.

Tener en cuenta las experiencias de los estudiantes es encontrarle sentido a su vida en este tipo de contexto - establecimientos penitenciarios -; las actividades de aprendizajes deben estar estructuradas de modo que tengan algo que ofrecerles, procurando situaciones en las que deba actualizar y utilizar autónomamente los conocimientos de los que se haya ido apropiando. Estas son solo algunas de las múltiples formas en que los profesores que trabajan en establecimientos penitenciarios ayudan al estudiante a construir significados sobre contenidos de diversa naturaleza.

El tercer principio: clima educativo adecuado teniendo en cuenta el escenario donde se desarrollen las acciones, para el tipo de contexto sobre el cual se desarrolla la intervención educativa en el presente trabajo. Hay que tener en cuenta varios aspectos, entre ellos tenemos que comprender y conocer las bases teóricas y metodológicas que desde perspectivas pedagógicas, psicológicas y sociológicas sustentan los procesos socio educativos en establecimientos penitenciarios, así como los marcos legales que permitan orientar la acción del profesor.

Una vez realizado este paso se tiene que diseñar, planificar, gestionar, desarrollar y evaluar diferentes programas socio educativos efectuados en el medio penitenciario para dirigir, gestionar y coordinar organizaciones, centros e instituciones socio educativas de acuerdo a las características del contexto penitenciario para poder afrontar con espíritu crítico 
los deberes y dilemas éticos que las nuevas demandas y formas de exclusión social de la sociedad del conocimiento plantean a la profesión del profesor y el estudiante en el centro penitenciario.

El cuarto principio: sistema de evaluación y auto evaluación diseñados correctamente. Es necesario analizarlos desde diferentes puntos de vista; la práctica de evaluar es tan antigua como la propia civilización, pero su estudio de forma profesional y científica es muy reciente. La evaluación es un eslabón importante del proceso de formación de los estudiantes. Con la evaluación de la modalidad a distancia para este tipo de contexto se nota un cambio por parte de estudiante al ser evaluado, cambios como independencia, iniciativas, control personal, conciencia del proceso de enseñanza-aprendizaje y sus resultados. Se pone en evidencia la concepción teórica de Young $(1971$, p. 8) en la que el conocimiento constituye una relación entre un agente y el entorno, teniendo en cuenta un concepto psicológico importante que es la motivación que poseen estos estudiantes adultos es más intensa, vivaz, profunda, determinada por las exigencias del medio donde desarrollan sus estudios.

La planificación de intervención educativa mediante la educación a distancia para los establecimientos penitenciarios dada las exigencias de la Universidad actual tiene que volcarse a la búsqueda de vías, métodos y técnicas para su constante desarrollo; para responder a este reto está la modalidad de educación a distancia y junto con ella la utilización de las tecnologías de la información y la comunicación (TIC), pues en este mundo de internacionalización hay factores que parecen trascender unos sobre otros siendo uno de ellos la explotación de la información. Todo esto va transformándose irremediablemente para que la educación superior en centros penitenciarios posea caracteristicas especificas que trasformen al estudiante para su vida en libertad.

La clave en la educación ya no será la cantidad de conocimientos aprendidos, sino la habilidad para usar el conocimiento y el know-how (saber cómo). La educación del siglo XXI deberá caracterizarse por su completa apertura ofreciendo posibilidades de aprendizajes a todos, desformalizándose en cuanto a requisitos académicos y de edad, enseñará en sus aulas, en sus laboratorios, en fábricas, empresas, centros penitenciarios, hogares, es decir, universalizándose y extendiéndose a toda la sociedad buscando saber, conocimiento, verdad e inclusión.

La educación a distancia es precisamente la llamada a utilizarse para emprender una educación acertada utilizando las TIC, sistema que surgió aproximadamente hace algunos 300 años cuando la educación por correspondencia era lo que primaba. Se trata de un nuevo modelo educativo donde intervienen un conjunto de procesos y recursos que le sirven al estudiante para aprender.

Pero antes de continuar fundamentando la validez de este modelo de enseñanza tendremos en cuenta en este trabajo lo que piensan algunas instituciones y autores sobre la educación a distancia. 


\section{DEFINICIONES DE INSTITUCIONES}

1) La combinación de educación y tecnología para llegar a su audiencia a través de grandes distancias es el distintivo del aprendizaje a distancia. Esto viene a hacer un medio estratégico para proporcionar entrenamiento, educación y nuevos canales de comunicación para negocios, instituciones educativas, gobiernos y otros públicos y agencias privadas. Con pronósticos de ser uno de los siete mayores desarrollos en el área de la educación la educación a distancia es algo crucial en nuestra situación geopolítica como un medio para difundir y asimilar la información de base global (Texas, University).

2) Educación a distancia es distribución de educación que no obliga a los estudiantes a estar físicamente en el mismo lugar con el profesor. Históricamente la educación a distancia significa estudiar por correspondencia. Hoy el audio, el video y la tecnología de la computación son modos más comunes de envio (The Distance Learning Resource Network - DLRN).

3) El programa de educación a distancia se ha concebido como un modelo de educación no formal que permite integrar a personas que por motivos culturales, sociales o económicos no se adaptan o no tienen acceso a los sistemas convencionales de educación. Se orienta a ofrecer opciones de capacitación con demanda en las economías zonales y regionales (Universidad de Uruguay).

\section{DEFINICIONES DE AUTORES}

1) El Dr. Roberto Aparici profesor de la Universidad Nacional de Educación a Distancia de España (Uned) plantea que la educación a distancia es un modelo democrático de enseñanza que permite que todo el mundo pueda acceder a él. Y ahora con el empleo de las nuevas TIC el acceso será aún más fácil.

2) La Dra. Luisa Noa Silverio, profesora de la Universidad de la Habana, nos dice que la educación a distancia requiere utilizar diversas vías y medios para establecer la comunicación educativa, ya que por sus objetivos y las características propias del sector de la población a que van dirigidos sus servicios es imposible emplear las formas presenciales tradicionales, el proceso enseñanza-aprendizaje, de forma espacialmente localizada y sincronizada temporalmente.

3) La Lic. Hilda Bustamante del Centro de Educación a Distancia de Colombia agrega que la educación a distancia implica un sistema de planificación del trabajo, mecanización, racionalización de los procedimientos, así como la adopción de instrumentos de supervisión parecidos a los métodos industriales para atender a miles de personas. Podriamos decir que el sistema masificado de educación a distancia es una consecuencia lógica de los esfuerzos por facilitar el acceso a la educación. 
Utilizando estas definiciones de instituciones y de algunos autores que han realizado estudios en la materia podemos concluir que la educación a distancia posee elementos definitorios como por ejemplo la separación entre el profesor y el estudiante durante la mayor parte del proceso educativo, pues a través de esta modalidad educativa han podido participar personas que antes por diversas razones (trabajos, distancia del centro de estudio, por mayoría de edad, privación de libertad) habían quedado excluidos del sistema permitiendo su actualización permanente. La autora del trabajo después de realizar estudios sobre la educación a distancia la define como un modelo educativo donde interviene un conjunto de procesos y, a su vez, aplicación de recursos que le sirven al estudiante para que aprenda de forma independiente.

La aplicación de las TIC en la educación a distancia ha generado tres formas de aprendizajes que debemos tener en cuenta para desarrollarlo: independiente, abierto y colaborativo. El estudiante posee la posibilidad de escoger qué aprender (contenido), dónde aprender (local de aprendizaje), cómo quiere aprender (métodos), cuándo aprender (dia, semana, hora) el ritmo en que desea aprender, a quién solicitar ayuda para profundizar los conocimientos y a cuál sistema de evaluación desea ser sometido.

Las universidades poseen la misión de desarrollar e integrar conocimientos y su vínculo con la sociedad constituye su misión más importante, pues a decir del ministro de Educación Superior Fernando Vecino Alegret en la Conferencia Magistral, en La Habana: "La Universidad en la construcción de un mundo mejor" con lo cual plantea que hoy hay que luchar por el acceso más pleno a las universidades convirtiéndose esto en una necesidad insoslayable, porque el mundo precisa de que un número creciente de hombres y mujeres que sean cada día más cultos, cada día más conscientes de que el orden mundial actual es sencillamente complejo y diverso, comunicado y elitista. Pero, continuaba el profesor, que esto no se logrará si este profesional no ha crecido como ser humano; más adelante puntualizó que "el papel de la Universidad con la comunidad se irá enriqueciendo por el incremento de los universitarios en la vida política, cultural y socioeconómica del pais" (VECINO ALEGRET, 2004). La planificación de todo ese proceso educativo, dada las exigencias de la Universidad actual, tiene que volcarse a la búsqueda de vías, métodos y técnicas para su constante desarrollo y para responder a este reto está la modalidad de educación a distancia, y junto con ella la utilización de las TIC, pues en este mundo de internacionalización hay factores que parecen trascender unos sobre otros siendo uno de ellos la explotación de la información. Todo esto va transformándose irremediablemente para que la educación superior a distancia posea un nuevo paradigma vinculado con el desarrollo humano, aspecto fundamental en las aspiraciones de cualquier tipo de sociedad en correspondencia con el pleno respeto de los derechos humanos de todas y todos sus miembros, de ahí que la educación a distancia en centros penitenciarios comparta estas aspiraciones buscando saber, conocimiento y verdad. 
La educación a distancia se está desarrollando en aras de satisfacer las actuales exigencias sociales y demandas de conocimientos de la población; se piensa que puede ocuparse de cosas adicionales en una forma más rápida y económica.

Desde inicios, este tipo de enseñanza tuvo que enfrentarse a la desconfianza de quienes veian en esta una oportunidad menor pero se ha comprobado que esto no es cierto; muchas instituciones y profesores que la utilizan tienen la idea de que las oportunidades ofrecidas por la educación a distancia superan los obstáculos que presenta el uso de la tecnología. De hecho muchos de los profesores de educación a distancia aceptan que la preparación que requieren los cursos ayuda a mejorar su desempeño como maestros, pues los retos que desempeña este modelo de educación son compensados por las oportunidades que ella presenta.

La educación a distancia representa un sistema flexible, dinámico y, por supuesto, atractivo con el empleo de las TIC en los procesos de enseñanza aprendizaje con un apoyo eficiente en el manejo de la información que determina estrategias diferentes a las tradicionales clases magistrales con presencia directa del alumno y el profesor. Ella logra reunir estudiantes de diferentes ambientes culturales, económicos, sociales y con variadas experiencias laborales y de conocimientos.

Con esta visión futurista los profesores consideran la concepción del aprendizaje como procesamiento y construcción de la información, en lugar de acumulación de la información, sobre la base del principio según el cual los conocimientos no se construyen, sino que se reconstruyen, para lo cual todos los profesores que trabajan con esta metodología ven exigidas sus formas de hacer docencia. Sin importar las temáticas de enseñanzas deberían convertirse en verdaderos facilitadores/as y estimuladores/as de la creatividad, así como en mediadores/as entre el conocimiento, la información y las personas que construyen sus nuevos conocimientos. Si estas transformaciones logran darse en el medio abierto, donde la persona es libre, en la que no le acontece en ese momento de su vida un procesamiento penal, representaría una transformación cotidiana en la vida de las personas privadas de libertad.

En Uruguay aún no se cuenta con la posibilidad de pensar que las personas logren construir aprendizajes a través de la educación a distancia, a pesar de que es el país con mayor conectividad en América Latina. En los centros penitenciarios uruguayos por el momento resulta impensable, no por un tema de posibilidad tecnológica, sino porque aún las concepciones de educación adquieren limitación para estos usuarios/as.

Si se tienen en cuenta los instrumentos internacionales de las Naciones Unidas sobre la protección de personas sometidas a detención o prisión, los centros penitenciarios no estarían separados del derecho a una política inclusiva, pues como establecimientos de reclusión y cumplimiento de las penas no pueden escapar a esa concepción de educación para todos/as.

Se debe conformar un ambiente educativo, en el que garantizándose las funciones de reclusión y custodia, se creen espacios interiores en los que sea posible el cumplimiento de este fin esencial de la institución con los resultados más beneficiosos posibles desde la privación de libertad para la vida en libertad. 
A la educación en centros penitenciarios no les es ajena la formación universitaria de algunas personas privadas de libertad, que posean actitudes y aptitudes para ello, pues la mejor respuesta a sus delitos no es otra que la enseñanza como presupuesto de educación y cultura. Valorado por varios autores, en cuanto a que la presencia de esta población reclusa y madura en las universidades con toda su experiencia resulta un ingrediente enriquecedor a la vida universitaria que no debe desaprovecharse.

En la aplicación de la modalidad de educación a distancia y el empleo de las TIC se puede desarrollar este tipo de educación pues ella permite la libertad de tiempo y lugar, una independencia al usuario/a para acercarse a otro tipo de construcción del conocimiento o actualizarse sin tener que estar obligado a cumplir porcentajes de asistencia, horarios o concurrir a sitios distantes. Todo esto bien aplicado auspicia en las personas una búsqueda del sentido de su existencia. El contacto social de las personas privadas de libertad con profesores/as universitarios generará una apertura de la cárcel hacia la universidad que por más pequeña que sea puede tratarse del comienzo que desestructure la idea del adentro y del afuera como dos sociedades distintas.

Quizá este desafío también cambie aspectos que las universidades deben plantearse, la formación del profesional y la profesional universitario/a en centros penitenciarios ofrece grandes posibilidades; será válido entonces aclarar en cómo la educación superior jugará un papel importante dentro del proyecto de vida del recluso, si tenemos en cuenta el planteamiento del psicólogo Ovidio D'Angelo Hernández cuando apunta a que el proyecto de vida no es solo un modelo ideal, conjunto de representaciones mentales sistematizadas sobre cuya base se configuran las actitudes o disposiciones teóricas del individuo, sino que para ejercer una dirección auténtica de la personalidad, este modelo debe tomar una forma determinada en la propia actividad social del individuo y en sus relaciones con otras personas.

Se podria resumir, entonces, que a partir de este planteamiento para la persona privada de libertad, en su proyecto de vida no se base solo en lo que quiere ser, sino en su disposición real y las posibilidades externas e internas para que pueda lograrlo. Un sentido de realidad, restando un tanto de pensamiento mágico y retomando el contacto con una forma de pensamiento creativo, pueden darle una forma precisa al curso de sus vidas, habilitando desarrollar un proyecto de vida teniendo en cuenta planes del tiempo futuro de las personas privada de libertad y su relación con las formas de empleo, relacionándolo con sus intereses y orientaciones valorativas. El hecho de sentir que se sabe, que se puede facilitar la preparación para la convivencia en libertad, al tiempo que limita en la medida de lo posible, el efecto negativo que provoca la encarcelación.

En la comprensión que la educación universitaria ofrece más posibilidades de opción de vida, recobrando la confianza en sí mismos, teniendo una motivación importante que los incluye socialmente, genera el sentimiento de que sus sueños también son relevantes y que otra vida es posible. 


\section{Educational intervention through distance education in penitentiary centers}

Abstract: This article presents an explanation of the importance of the principles of intervention and educational approach for the model of distance higher education in prisons, since they are not alien to university education, considering that the presence of this population reclusive and mature in the universities with all their experience can be a valuable enriching ingredient of university life that should not be missed.

Keywords: Long distance education. Penitentiary centers. Education. Higher education. Intervention.

\section{REFERENCIAS}

ÁLVAREZ DE ZAYAS, C. M. Fundamentos teóricos de la dirección del proceso docente-educativo en la Educación Superior Cubana. La Habana: Félix Varela, 1989.

ÁlVAREZ DE ZAYAS, C. M. Pedagogía como ciencia. La Habana: Editorial Félix Varela, 1998.

ARALLO VARELA, S. R. El uso de las nuevas tecnologías: la teleformación. Málaga: Universidad de Málaga, 1999.

BAENA MARTíN, N. El futuro de la multimedia. Técnicas de la comprensión de la información digital. Transformada de Wanelet. Aplicaciones. In: CONGRESO NUEVAS TECNOLOGÍAS DE LA INFORMACIÓN Y LA COMUNICACIÓN PARA LA EDUCACIÓN, 1997, Málaga. Málaga, 1997.

BALLESTEROS REGEÑA, C. Educación y nuevas tecnologias: un diálogo necesario y una realidad evidente. Revista de Medios y Educación, n. 22, p. 5-39, ene. 1997.

BENITEZ MOLINA, A. ¿Sistema penitenciario en Centroamérica o bodegas humanas? San José de Costa Rica: Codehuca, 1999.

CABERO, J.; DUARTE, A.; BARROSO, J. La piedra angular para la incorporación de los medios audiovisuales, informáticos y nuevas tecnologias en los contextos educativos: la formación y el perfeccionamiento del profesorado. Revista Electrónica de Tecnología Educativa, n. 8, nov. 1997.

CABERO ALMENARA, J. Nuevas tecnologías, comunicación y educación. Palma de Mallorca: Universidad de Islas Baleares, 1996.

CABERO ALMENARA, J. Fuentes documentales para la investigación audiovisual, informática y nuevas tecnologías de la información y documentación. Revista Electrónica de Tecnología Educativa, n. 8, nov. 1997.

COMUNICACIÓN, TECNOLOGÍA Y EDUCACIÓN. Oviedo: Universidad de León, 1998. FUENTES GONZÁLEZ, H. C. et al. Modelo holistico configuracional de la didáctica. Centros de Estudios de Educación Superior Manuel F. Grean. Santiago de Cuba: Universidad de Oriente, 1998. 
GARCÍA PASCUAL, E. ¿Qué hace un pedagogo como tú en una multimedia como este? Aventuras y desventuras para iniciarse en la multimedia desde un centro de formación inicial del profesorado. Zaragoza: Universidad de Zaragoza, 1997.

GISBERT CERVERA, M. El docente y los entornos virtuales de enseñanza-aprendizaje. Cuadernos de Documentación Multimedia, 1997. Disponible en: <http://www.ucm.es/info/multidoc/ revista>. Acceso en: 28 ago. 2018.

D'ANGELO HERNÁNDEZ. Proyecto de vida como categoría básica de la interpretación de la identidad individual y social. Revista Cubana de Psiocología, v. 17, n. 3, 2000.

LECCIONES de filosofía marxista-leninista. Tomo I-II. La Habana: Editorial Félix Varela, 2003.

LORENZO MOLEDO, M. La delincuencia femenina en Galicia. La intervención pedagógica. Galicia: Junta de Galicia, 1997.

MARTí GONZÁLEZ, Y. Educación y nuevas tecnologias. Una relación creativa. Ponencia. cd-r. Convención Universidad de Granma, 2003.

MARTí PÉREZ, J. Ideario pedagógico. La Habana: Imprenta Nacional, Ministerio de Educación, 1961.

MONTESINOS LÓPEZ, A. El uso didáctico del correo electrónico en la enseñanza superior. Valencia: Universitat Politécnica de Valencia, 1997.

NOA SILVERIO, L. ¿Cómo hacer de internet un real instrumento de trabajo intelectual? La Habana: Facultad de Educación a Distancia, 2002.

NÚÑEZ JOVER, J. La ciencia y la tecnología como procesos sociales. Lo que la educación científica no debería olvidar. La Habana: Editorial Félix Varela, 1999.

PÉREZ, R. Actitudes del profesorado hacia la incorporación de las nuevas tecnologías de la comunicación en educación. Oviedo: Universidad de Oviedo, 1997.

RUIZ SOLER, M. Enseñanza asistida por ordenador: ¿cuál es su punto débil? Málaga: Universidad de Málaga, 1999.

VECINO ALEGRET, F. Conferencia Magistral. In: Congreso Universidad 2004,. feb. 2004, La Habana, Cuba.

VIEGAS FERNÁNDEZ, J. La educación que necesitamos. Revista Temas, n. 31, p. 4-17, oct./dic. 2002.

YOUNG, M. F. D. Knowwledge and control. London: Collier-Macmillan, 1971.

ZAPATA, 0. El grupo en los procesos de enseñanza-aprendizaje en la educación superior. Revista Reencuentro, v. 22, p. 45,1999. 